\title{
Klinikpartnerschaften - ein Weg zur Verbesserung der Patientenversorgung
}

C. Schäfer

Korrespondenz:

Dr. med. Christian Schäfer

Klinik Sonnenhalde

Gänshaldenweg 28

CH-4125 Riehen

c.schaefer@sonnenhalde.ch
«Es ist hier gängige Praxis, die Leute einfach unter Kontrolle und in Verwahrung zu halten. Die Insassen, die ganz offensichtlich von der Gesellschaft verstossen worden sind, müssen ohne jegliche Beschäftigung auskommen, es gibt keinerlei Hoffnung für sie. Sie werden mit ihresgleichen zusammengepfercht ... Nichts gibt ihrem Leben Sinn.»

Dr. med. M. Myers, Psychiaterin, die 2002 mit ai bulgarische Psychiatrien und Heime besuchte

Als wir im Jahr 2002 Kontakt mit der psychiatrischen Klinik Zerowa Korija zum Sondieren einer Partnerschaft aufnahmen, war uns diese Aussage nicht bekannt. Wir wussten wie so viele Westeuropäer sehr wenig von Bulgarien. Lediglich der beginnende Massentourismus an die Schwarzmeerküste wegen der günstigen Preise war uns bekannt.

«Wir», die psychiatrische Klinik Sonnenhalde, eine Privatklinik mit sechzig offenen Betten, hatten als eines unserer Projekte für die nächsten Jahre eine Partnerschaft mit einer osteuropäischen Klinik zum Ziel.

Der erste Eindruck war ernüchternd: Das Schweizer Team besuchte eine Klinik etwa 15 Ki- lometer von der Provinzhauptstadt Veliko Tarnovo in einem kleinen Dorf ungefähr in der Mitte Bulgariens. Ein öffentlicher Nahverkehr existierte praktisch nicht. Die Strassen waren in einem erbärmlichen Zustand. Wir trafen eine Klinik mit etwa 150 Patienten, acht Ärzten und etwa 30 Pflegenden an. Die Klinik befand sich baulich in einem sehr schlechten Zustand, die sanitären Anlagen auf den vier Abteilungen wagten wir nicht zu fotografieren. Die Patienten lebten meist in Achtbettzimmern, die ausser den Betten nichts anderes aufnehmen konnten.

«Aufenthaltsraum» war der Abteilungsgang und eine Erweiterung des Ganges mit einem Fernseher. Diese Nische war gleichzeitig Essraum. Aufgrund seiner Grösse mussten die Patienten dort schichtweise ihr Essen einnehmen. Für die Patienten gab es so gut wie keine therapeutischen Massnahmen. Die meiste Zeit des Tages lagen sie im Bett. Die Kleidung bestand aus Klinikpyjamas, die sie Tag und Nacht trugen.

Der Tagessatz für medikamentöse Versorgung und Löhne für das Personal betrug etwa 5 Euro. Die Dokumentation war mangelhaft, und eine regelmässige Weiterbildung fand nicht statt.

\section{Partenariat entre cliniques - un chemin pour améliorer les soins}

Un partenariat existe depuis 2002 entre la clinique psychiatrique Sonnenhalde à Riehen et la clinique psychiatrique Zerowa Korija en Bulgarie centrale. La clinique bulgare ne pouvait alors compter que sur elle-même et luttait avec des moyens extrêmement restreints pour faire vivre ses quelque $150 \mathrm{pa}$ tients. Les installations sanitaires se trouvaient dans un état lamentable, la nourriture distribuée aux patients était rationnée et les possibilités thérapeutiques étaient inexistantes, hormis l'administration de médicaments. Avec le soutien financier de la DDC, la clinique Sonnenhalde entreprit de collaborer, sous forme partenariale, avec la clinique bulgare pour améliorer la situation de I'hôpital et notamment celle des patients.
Après cinq ans de partenariat, la clinique est dotée de vergers et d'un grand jardin potager ainsi que d'une porcherie. La thérapie par le travail dans les champs profite de surcroît aux patients. La formation continue donnée au personnel lui a permis de passer d'une psychiatrie de surveillance à une psychiatrie activante. De nouvelles thérapies ont été introduites et la documentation a été grandement améliorée. Les chambres et les installations sanitaires ont été rénovées et l'atelier créatif est devenu un exemple pour les cliniques psychiatriques de Bulgarie. La clinique suisse a aussi largement profité de ce partenariat. Les différents groupes professionnels ont appris, en particulier, à mieux se comprendre entre eux. 


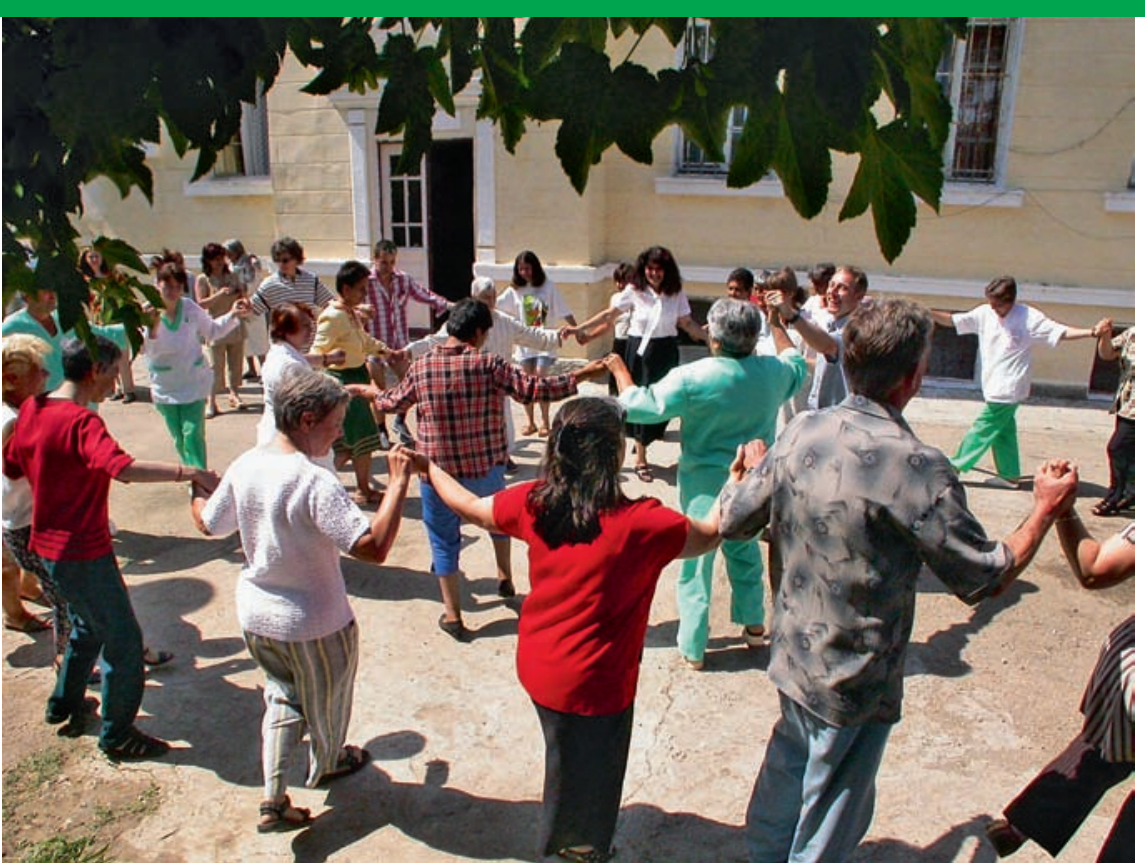

Patienten und Personal beim gemeinsamen Tanzen im Hof der Klinik ...

Ernüchterung auch bezüglich der Anliegen: So berichtete die Chefärztin der Klinik in Zerowa Korija, dass sie einige Zeit zuvor nicht gewusst hatte, wie sie genügend Nahrung für ihre Patienten organisieren konnte. Jeder Winter sei eine Herausforderung bezüglich der Heizung.

In einem Vertrag wurden nach weiteren $\mathrm{Ab}$ klärungsgesprächen vier Ziele im Rahmen der Partnerschaft festgelegt:

1. Verbesserung der Versorgung des Krankenhauses;

2. Aktivierung der Patienten;

3. Weiterbildung der Ärzte und der Pflegenden;

4. Verbesserung des Images des Krankenhauses.

Die Partnerschaft wurde schon zu Beginn zeitlich begrenzt (Eintritt Bulgariens in die EU) und die Notwendigkeit der Nachhaltigkeit der Projekte als primäres Ziel gesetzt. Finanziell wurde das Projekt durch die DEZA mitgetragen. Zweimal jährlich flog ein Schweizer Team in die Klinik, um dort zu schulen oder Projekte anzustossen. Bei den jährlichen Gegenbesuchen hospitierten die bulgarischen Gäste auf den Abteilungen der Schweizer Klinik. Die einzelnen Projektziele wurden parallel in den ersten Jahren begonnen und bei den Besuchen einzelne Schwerpunkte gesetzt.

\section{Verbesserung der Versorgung}

Die schlechte Versorgung an Nahrungsmitteln war zu Beginn der Partnerschaft ein gerade für das Schweizer Team psychisch belastendes Thema: Die Patienten bekamen drei- bis viermal im Jahr Fleisch zu den täglichen Mahlzeiten, sonst gab es meist Brei, Gemüse und Brot. Viele Patienten verbrachten Jahre in der Klinik, da
Nachfolgeeinrichtungen in Bulgarien noch unbekannt sind und Familien sich von psychisch Kranken zurückziehen.

Bedingt durch die ländliche Lage, besass die Klinik grössere Ackerflächen und alte Ställe, die in sozialistischen Zeiten noch in Betrieb waren, nach der Wende («before the change» und «after the change» sind in Bulgarien beliebte Zeitangaben) aufgrund von Geldmangel nicht mehr weitergeführt werden konnten.

Hier unterstützten wir den Kauf von Schweinen, die Neuanlage der Ackerflächen und die Anpflanzung von etwa 200 Obstbäumen. Die bulgarische Klinik finanzierte die Einstellung eines Gärtners. Weitere Arbeitskräfte mussten nicht eingestellt werden, da gleichzeitig im Rahmen einer neu initiierten Arbeitstherapie Patienten auf dem Acker und bei der Versorgung der Schweine mithalfen.

Der anfängliche teure Einkauf von Schweinefutter konnte im Lauf der Jahre durch den Anbau von Luzernen deutlich reduziert werden. Die Patienten bekommen momentan drei- bis viermal wöchentlich Essen mit frischen Gemüse und Fleisch. Zusätzlich konnten durch einmalige finanzielle Zuschüsse das Essgeschirr, die Teller und die Stühle in den Aufenthaltsräumen neu angeschafft werden.

\section{Aktivierung der Patienten}

Eine Aktivierung der Patienten als therapeutische Massnahme wurde zu Beginn der Partnerschaft in der Klinik nicht durchgeführt. Der Tag war ausschliesslich durch die Mahlzeiten gegliedert. Die medikamentöse Behandlung war einzige Säule der Therapie.

Nach zweimaligen Besuchen einer Ergotherapeutin der Schweizer Klinik konnte ein Kreativatelier und ein Nähatelier, in dem die Patienten ihre Kleidung für den Austritt herrichteten, eingerichtet werden.

Ebenfalls nach mehrmaliger Motivation unsererseits entstand ein Sportplatz auf dem Klinikgelände, eine Waschmaschine wurde auf der offenen Therapieabteilung angeschafft, und die regelmässige Arbeitstherapie im Gemüsegarten und bei den Schweinen war ein weiterer wichtiger Schritt.

Problematisch gestaltete sich die therapeutische Ausrichtung der Aktivierung: Anfangs ging es vor allem um die «Produktion» von Werken aus dem Kreativatelier, und in den Besprechungen über die Landwirtschaft wurden dem Schweizer Team stolz die Zahlen über die «Ernteerfolge» gezeigt. Erst allmählich (eine mitgebrachte Videoaufnahme eines Therapeutenrapportes war dabei wertvoll) kam es zu einer Änderung der 
Denkstrukturen und zu einer therapeutischen Dokumentation und Beobachtung der Patienten.

Ein weiterer wichtiger Schritt zur Aktivierung und Aussenorientierung der Patienten war die Einrichtung einer Tagesklinik auf dem Gelände der Klinik. Hier nahmen stabile Patienten aus den Abteilungen am Programm teil, und schon entlassene Patienten kamen mit dem Klinikbus, der am Morgen das Personal zur Arbeit brachte, aus der Stadt, um sich weiter zu stabilisieren.

\section{Weiterbildung des Personals}

Bei jedem Besuch in unserer Partnerklinik wurden sowohl Ärzte als auch das Pflegepersonal weitergebildet. Dabei kamen uns die im Vorfeld der EU-Aufnahme gemachten Direktiven der Regierung zu Hilfe.

Problematisch war der Umgang des gesamten Personals mit den Patienten, der zusammengefasst euphemistisch als «kustodial» bezeichnet werden konnte. So wurden die Auflagen der Regierung, eine differenzierte Dokumentation einzuführen, das Recht auf Anhörung vor Gericht und die Vorgabe grundlegender Patientenrechte, zuallererst als Eingriff in die Therapiefreiheit und als zusätzliche Arbeit gewertet.

Vor allem beim Pflegepersonal trat im Laufe der Zeit ein Wandel des Denkens ein, da es durch die neue Dokumentationspflicht und die eingeführten interdisziplinären Rapporte zu einer Auf- wertung des Pflegeberufes kam. Auch wurde nach einiger Zeit die vermehrte Strukturierung des Arbeitsablaufes (Patientenrunden, Spiele auf der Abteilung, Bezugspersonenarbeit) weniger als zusätzliche Arbeit und mehr als Arbeitserleichterung gesehen.

Schwieriger war es, die Ärzte in den Umdenkungsprozess mit einzubeziehen, da sie sich in ihrer Machtfülle beschnitten sahen. Hier standen vor allem Weiterbildungen im psychotherapeutischen Vorgehen neuerer Erkrankungen (Essstörungen, Internetsucht) sowie der Umgang mit den Patienten im Sinne der Milieutherapie im Vordergrund.

\section{Imageverbesserung}

Die Lage der psychiatrischen Patienten war 2002 in Bulgarien stark stigmatisiert. Jeder Patient, der psychiatrisch behandelt wurde, wurde gleichzeitig zentral in der Provinzhauptstadt registriert. Gleichzeitig war diese Registrierungsstelle Anlaufstelle für alle Einwohner, die zum Beispiel heiraten wollten, die Arbeitsstelle wechselten oder für sich den Führerschein beantragten. Datenschutz war nicht gegeben, und Psychiatriepatienten wurden als Menschen zweiter Klasse behandelt.

Gleichzeitig war das Interesse der Medien (im Gegensatz zur Schweiz) an der Partnerschaft enorm: Praktisch bei jedem Aufenthalt in unserer Partnerklinik gab es Interviewanfragen von

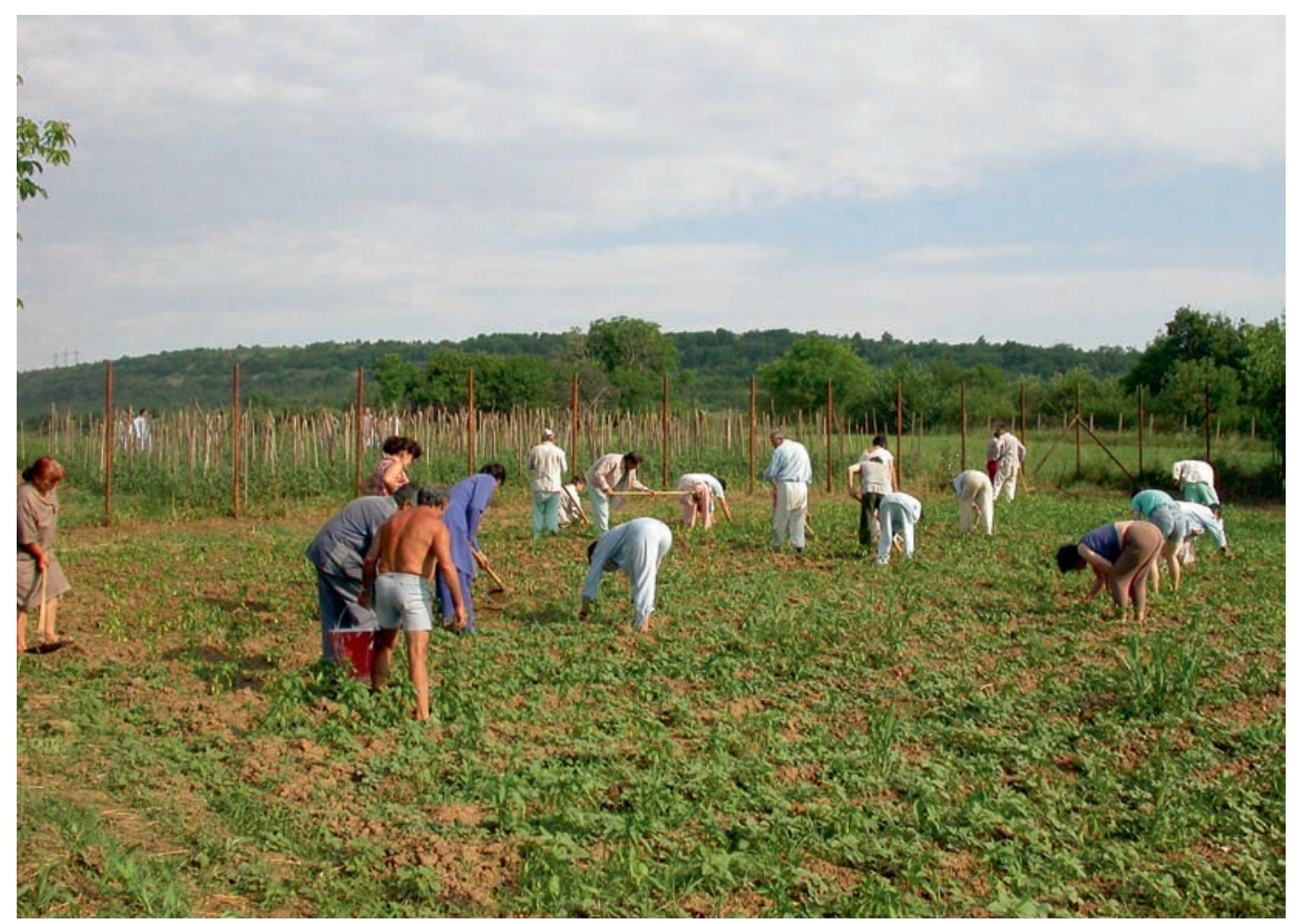

... und beim gemeinsamen Arbeiten auf dem Feld. 
lokalen Zeitungen, den örtlichen Radiostationen und dem Fernsehen. Die Presse berichtete ausführlich und wohlwollend über das Projekt. Die allmähliche Entstigmatisierung und das gewachsene Selbstbewusstsein unserer Partnerklinik machten sich im Laufe der Partnerschaft durch die Herausgabe eines eigenen Klinikprospektes und das Einrichten einer Homepage bemerkbar.

\section{Fortschritte}

«Die fünf Jahre der Partnerschaft waren für uns sehr wichtig. Ohne die Klinik Sonnenhalde hätten wir nicht gewusst, wie wir diese Zeit hätten meistern können. Jetzt hoffen wir mit Hilfe der EU weiter gehen zu können.» Dieses Statement von Chefärztin Dr. med. Genova zum Abschluss der Partnerschaft spiegelt die doch trotz aller Probleme auch hoffnungsvolle Einstellung der Klinik wider.

Nach fünf Jahren Partnerschaft hat sich die zuvor kustodiale Psychiatrie zu einer zeitgemässen Psychiatrie mit einer durchgängigen Aktivierung und Rehabilitationsorientierung entwickelt. Vielfache Fortbildungen auf allen Ebenen halfen mit, diese Grundhaltung in der Ärzteschaft und in den Pflegeteams fest zu verankern. Ergänzt wird diese grundlegende Veränderung auch durch eine Verbesserung der gesamten Lebensverhältnisse, die durch das Landwirtschaftsprojekt und die Schweinezucht einen Aspekt der Selbstversorgung erhalten haben.

Neue Therapien wie regelmässiges PMR wurden eingeführt und die Dokumentation erheblich verbessert. Gerade im Bereich der aktivierenden Pflege wurden milieutherapeutische Strukturen der Schweizer Klinik auf die Klinik in Zerowa Korija adaptiert.
Ein Sportplatz entstand auf dem Klinikgelände, und eine Tagesklinik begann zu arbeiten. Die Zimmer und sanitären Anlagen wurden saniert, und das Kreativatelier hat Beispielcharakter für die psychiatrischen Kliniken Bulgariens bekommen. Die Klinik wurde wiederholt im Fernsehen als auch in den Zeitungen positiv erwähnt.

Durch die Partnerschaft wurden weitere Personen und Einrichtungen motiviert, die Klinik zu unterstützen: So wurde die Telefonanlage der Klinik durch Schweizer Privatinitiative neu aufgebaut, und eine benachbarte psychiatrische Klinik, die von der Partnerschaft erfahren hatte, spendete ein Auto für Patiententransporte. Auch konnten die Gartensitzbänke durch eine private Spende erneuert werden.

Was sind die Fortschritte für die Klinik Sonnenhalde? Sicherlich sind diese nicht in sichtbaren äusseren Projekterfolgen messbar. Dennoch möchte ich behaupten, dass mit den Einsätzen das Verständnis für die unterschiedlichen Berufsgruppen innerhalb der Klinik gewachsen ist. Unterschiedliche Therapiestrategien der einzelnen Abteilungen konnten diskutiert werden, und das Verständnis und die Hochachtung füreinander wuchsen. Auch Dankbarkeit für das hier schon Erreichte und die Relativierung von Wünschen waren ein Ergebnis. Manch einer besucht dafür teure Workshops und Supervisionen.

Wir werden unsere ehemalige Partnerklinik durch halbjährliche Besuche weiter beratend und supervidierend begleiten und vertrauen darauf, dass Zerowa Korija nun auch mit Hilfe der EU ihren Weg gehen kann. 